\title{
Dynamics of Domain Walls in Pattern Formation with Traveling-Wave Forcing
}

\author{
S. Rüdiger, ${ }^{1}$ J. Casademunt, ${ }^{2}$ and L. Kramer $^{3}$ \\ ${ }^{1}$ Institut für Physik, Humboldt-Universität zu Berlin, Newtonstraße 15, 12489 Berlin, Germany \\ ${ }^{2}$ Facultat de Física, Universitat de Barcelona, Avenida Diagonal 647, 08028 Barcelona, Spain \\ ${ }^{3}$ Universität Bayreuth, Universitätstraße 30, D-95448 Bayreuth, Germany
}

(Received 14 July 2006; published 9 July 2007)

\begin{abstract}
We study dynamics of domain walls in pattern forming systems that are externally forced by a moving space-periodic modulation close to 2:1 spatial resonance. The motion of the forcing induces nongradient dynamics, while the wave number mismatch breaks explicitly the chiral symmetry of the domain walls. The combination of both effects yields an imperfect nonequilibrium Ising-Bloch bifurcation, where all kinks (including the Ising-like one) drift. Kink velocities and interactions are studied within the generic amplitude equation. For nonzero mismatch, a transition to traveling bound kink-antikink pairs and chaotic wave trains occurs.
\end{abstract}

The effects of external forcing on pattern forming systems exhibit fascinating nonlinear behavior from a fundamental point of view and at the same time provide a valuable tool for the control of pattern forming systems. While the cases of purely temporal [1-3] and spatial forcing [4-6] have been considered for many years, the spatiotemporal modulation of control parameters has been introduced only recently [7-13]. Simultaneously, both fundamental questions [14] and interesting applications of pattern control have arisen for possible information processing devices based on nonequilibrium patterns $[15,16]$.

In the simplest case of a spatiotemporal forcing, using the form of a traveling wave, one allows for a periodic dependence on both space and time. The consequences of spatial resonance of such a forcing with a Turing-like mode were studied in terms of the frequency, or velocity, $\omega$, of the traveling-wave forcing and the deviation $q$ from exact spatial resonance. A central finding was the occurrence of kinks or domain walls [7], which, similar to the case of purely spatial forcing [5], mediate the competition between the inherent and imposed wavelength.

In this Letter we study how a mismatch $q$ and the motion of the forcing affect domain walls. We show that a combination of both effects, studied in the exemplary case of 2:1 resonance, provides a new scenario of complex spatiotemporal dynamics. Beginning with exact spatial resonance, $q=0$, an illuminating analogy with the resonance of a subharmonic temporal forcing of an extended oscillatory system appears. For the latter system it is well known that the resonance generates stable walls and a transition between Ising and Bloch walls. The motion of the forcing pattern-playing the role of the frequency detuning in oscillatory systems - endows the system with nongradient dynamics. This transition is called nonequilibrium IsingBloch (NIB) transition [17-21].

The effects of a spatial resonance mismatch are more difficult to analyze. While for large bifurcation parameter $\mu$ (or, equivalently, for small forcing) the effect of the mismatch becomes negligible and a phase approximation can be used [11], we here focus on intermediate values of $\mu$. We employ numerical continuation methods to calculate kink solutions and show that the mismatch $q$ renders the NIB bifurcation imperfect. A mismatch not only shifts the existence range of certain kinks, but also breaks their chiral symmetry, which results in inherently different dynamical properties, with, for instance, different drift velocities for the three types of kinks. The complexity of kink interaction characteristic for the spatial forcing with wave number competition (oscillatory interaction and locking of Shilnikov defects, which is at the root of purely spatial chaos [22] ) is now present but with nontrivial dynamical properties. In particular, we observe that spatially chaotic locked states turn into chaotic wave trains, propagating with a nontrivial velocity.

Our starting point is a generic amplitude equation for the slow envelope $A$ of a pattern $A(x, t) \exp \left[i\left(k_{c}+q\right) x-\right.$ $i \omega t / 2]+$ c.c.. The system, if unforced, undergoes a stationary, supercritical bifurcation with critical wave number $k_{c}$, and is subjected to a traveling-wave modulation of the control parameter of the form $\cos \left(k_{f} x-\omega t\right)$, close to 2:1 resonance, that is, $k_{f}=2\left(k_{c}+q\right)$. Here $q$ denotes a (small) wave number mismatch. The corresponding amplitude equation with proper normalization reads $[5,11]$

$$
\dot{A}=\left(\mu-q^{2}+\frac{i \omega}{2}\right) A-|A|^{2} A+A^{*}+\frac{\partial^{2} A}{\partial x^{2}}+2 i q \frac{\partial A}{\partial x},
$$

where the star denotes complex conjugation. With $q=0$, Eq. (1) coincides with that for an oscillatory extended system under subharmonic forcing, where $\omega$ plays the role of frequency detuning. Therefore, sufficiently near threshold, the case $q=0$ can be exactly mapped to that. Homogeneous and localized solutions of this system were extensively studied in recent years [3]. The mismatch $q$, 
however, introduces a new symmetry breaking, which has no analogue in the oscillatory system.

We first look for homogeneous solutions of (1). These fixed point solutions represent periodic locked patterns of wave number $k_{c}+q=k_{f} / 2$ moving with velocity $\omega / k_{f}$ in the laboratory frame. To study the stability of the fixed point $A=0$ we distinguish the cases $|\omega|<2$ and $|\omega|>2$. If $|\omega|<2$ the zero solution loses stability at a pitchfork bifurcation at $\tilde{\mu}=-\sqrt{1-\omega^{2} / 4}$ (where we have introduced $\tilde{\mu}=\mu-q^{2}$ ). Writing $A$ in polar variables $A=$ $R \exp i \Theta$, the two stable fixed points that bifurcate are $A_{h}^{ \pm}=R_{h}^{ \pm} \exp i \Theta_{h}^{ \pm} \quad$ with $\quad \Theta_{h}^{+}=\frac{1}{2} \arcsin \omega / 2, \quad \Theta_{h}^{-}=$ $\pi+\frac{1}{2} \arcsin \omega / 2$, and $\left(R_{h}^{ \pm}\right)^{2}=\tilde{\mu}+\sqrt{1-\omega^{2} / 4}$.

If $|\omega|>2$ the trivial solution becomes Hopf unstable at $\tilde{\mu}=0$ with Hopf frequency $\sqrt{\omega^{2} / 4-1}$. Nonzero fixed points do not exist and the system will be time dependent or nonhomogeneous.

Next we study domain walls for $|\omega|<2$, which connect the two stable fixed points. For $q=0$ one finds two qualitatively different types of wall solutions [17]. Let us first look at the case $\omega=0$. For small $\tilde{\mu}$ one finds Ising walls for which the amplitude crosses the origin [see Fig. 1(a)]. This is not the case for Bloch walls [Fig. 1(c), solid lines]. A pitchfork bifurcation between Ising and Bloch walls occurs at a critical $\tilde{\mu}_{c}=3$ (see Fig. 2). Nonzero $\omega$ does not destroy the basic structure of Ising and Bloch walls and their transition, but it shifts the location of fixed points [Fig. 1(b) and 1(d)] and introduces nongradient dynamics,
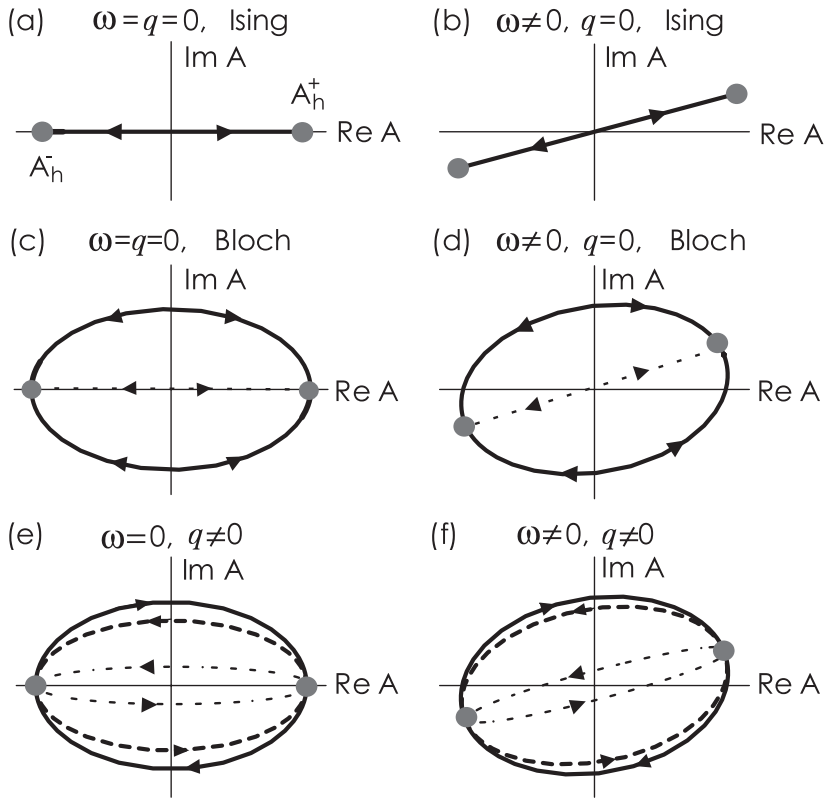

FIG. 1. Projections of domain walls into the complex plane of $A$. The kinks approach fixed points (gray dots) in the limit of infinite $|x|$. Dotted curves correspond to unstable solutions. The dashed and dotted curves in (e), (f) are kinks created in the saddle node bifurcation of the imperfect Ising-Bloch transition. conferring the inherently nonequilibrium character of the NIB transition [17,23].

For $q \neq 0$ the only types of walls are Bloch walls in the sense that the amplitude is always nonzero [Fig. 1(e) and 1(f)]. The introduction of a mismatch acts as an imperfection to the pitchfork bifurcation between Ising and Bloch walls. To calculate the corresponding bifurcation diagram we used numerical continuation by the HOMCONT routine of AUTO, XPPAUT [24]. This method obtains kinks as heteroclinic connections in the spatial ordinary differential equation derived from Eq. (1) [25].

For $\omega=0$ and $q=0.1$ the three branches of kinks connecting $A_{h}^{+}$with $A_{h}^{-}$are shown by solid lines in Fig. 2. For small $\tilde{\mu}$ only one kink exists. We denote this branch (and the related branch of kinks which connect $A_{h}^{-}$ to $A_{h}^{+}$) by $B 1 . B 1$ is generated at $\tilde{\mu}=-1$ together with the fixed points $A_{h}^{ \pm}$. We note that all $B 1$ kinks possess the same unique chirality. The branches $B 2$ and I are created in a turning point at $\tilde{\mu} \approx 5$ leading to the kinks projected in Fig. 1(e). Numerical simulation of the full Eq. (1) shows that the branches $B 1$ and $B 2$ are stable and the branch $I$ is unstable. Similar behavior is found for any nonzero $|\omega|<$ 2.

As shown by the dashed line in Fig. 4 the location of the turning point is shifted to larger values of $\tilde{\mu}$ for increasing $q$. Intuitively it is clear that for increased spatial mismatch $q$ one of the two Bloch branches (B2) is shifted to higher $\tilde{\mu}$. $B 1$ reduces the wave number of the underlying pattern from $k_{c}+q$ to smaller values by reducing the phase by $\pi$. It will then be closer to the natural wave number $k_{c}$. On the other hand, $B 2$ increases the wave number beyond $k_{c}+$ $q$ such that for large $q$ this wave number becomes increasingly unfavorable.

If $\omega \neq 0$, all walls, except for Ising walls for $q=0$, break spatial reflection symmetry and consequently drift [17]. In general, the drift velocity of isolated walls depends in a nontrivial way on $\mu, \omega$, and $q$. The absolute velocities

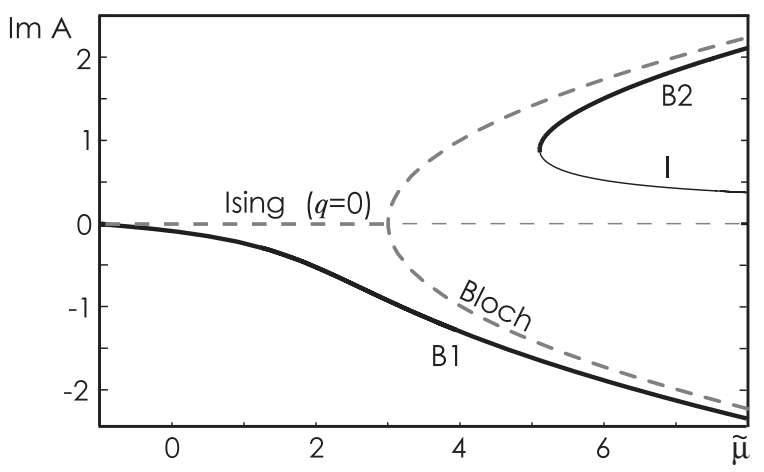

FIG. 2. Plot of the NIB transition ( $q=0$, dashed lines) and the imperfect transition ( $q=0.1$, solid lines) for $\omega=0$. The graphs show the imaginary component of $A$ at the center of each kink that connects $A_{h}^{+}$with $A_{h}^{-}$. Thick lines correspond to stable kinks. 
of the three branches of kinks for $\tilde{\mu}=8$ and $q=0.1$ are shown in Fig. 3. The unstable I branch has the smallest velocity. We also note that $B 2$ and I kinks propagate in a direction opposite to $B 1$ kinks.

Several analytical approximations were used to determine the drift velocity $[11,17,23]$. Here we mention the result for the phase regime $\left[\mu \gg\left(1, q^{2}\right)\right]$. In this case the drift velocity $v$ is a function of $\omega$ only. A nontrivial characteristic curve $v(\omega)$, similar to that of $B 1$ in Fig. 3, can be obtained as the location of a heteroclinic connection in the phase equation [11]. For small $\omega$ this curve can be approximated linearly by $v=\omega \pi / \sqrt{32}$ (dashed line).

Next we consider interactions of traveling kinks. In numerical simulations it is easy to find bound pairs and aperiodic arrays of kinks of equal chirality (see supplementary online material [26]). Counterpropagating kinks (i.e., kinks of opposite chirality) annihilate each other. As discussed above, counterpropagating kinks only exist above the kink saddle node to the right of the dashed curve in Fig. 4.

To find the existence range of stable kink pairs we determined the asymptotic spatial decay of kinks, which dominates the interaction for distant kinks [22]. The decay is given asymptotically by the spatial eigenvalues of the linearization around fixed point. For complex eigenvalues the interaction is repulsive or attractive depending on the distance of kinks.

To obtain the eigenvalues we linearize the ordinary differential equations obtained from (1) and a travelingwave ansatz, $A(x, t)=A(x-v t)$. Here $v$ is the velocity of the defect solution. For the fixed points $A_{h}^{ \pm}$the characteristic equation with eigenvalues $p$ reads:

$$
\begin{aligned}
& p^{4}-2 v p^{3}+\left(4 q^{2}-2 \tilde{\mu}-2 \sqrt{4-\omega^{2}}+v^{2}\right) p^{2} \\
& +\left(2 \omega q-2 v \tilde{\mu}-4 v q^{2}+2 v \sqrt{4-\omega^{2}}\right) p \\
& +4-\omega^{2}+2 \sqrt{4-\omega^{2}} \tilde{\mu}=0 .
\end{aligned}
$$

This equation has been evaluated using the velocity $v$ obtained from numerical continuation. To locate transitions of eigenvalues from real to complex values for fourth order equations, one can conveniently use the discriminant of its cubic resolvent.

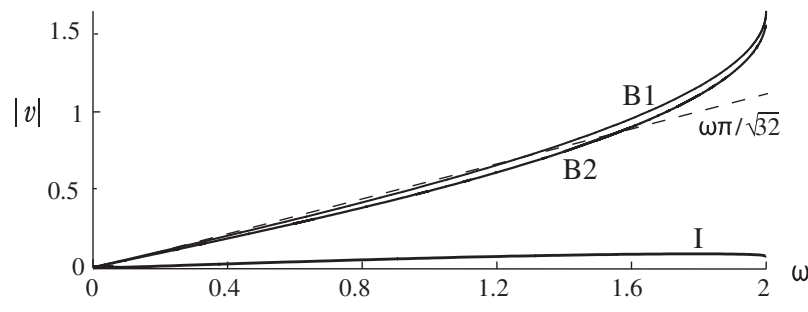

FIG. 3. Velocity of all three types of kinks for $\tilde{\mu}=8$ and $q=$ 0.1 . The two stable branches $B 1$ and $B 2$ end in a saddle node at $\omega=2$, where $|v|=1.652$ and $|v|=1.592$, respectively.
For $\omega=0$ two pairs of eigenvalues are always related by an opposite sign: $\lambda_{1}, \lambda_{2},-\lambda_{1},-\lambda_{2}$. Consequently, all eigenvalues become complex at the same location-the thick line in Fig. 4. For comparison, the stable distance of defects was determined by numerical simulations of Eq. (1) and is shown for $\mu=1$ in Fig. 5 (stars; diamonds show the minimal distance of two kinks to repel each other). No bound states were found for $q$ below 0.28 , in accordance with the results in Fig. 4.

For nonzero $\omega$ the coupling of eigenvalues is lost and eigenvalues can, in general, have different absolute values. Figure 4 contains results for $\omega=0.1$. We found two curves of zero discriminant (thin solid and dotted lines) indicating a transition from real to complex eigenvalues. Now pairs of eigenvalues become complex at these two different locations. Between the thin solid and the dotted curves two complex and two real eigenvalues exist. Above the dotted curve all four eigenvalues are complex.

We have repeated the numerical simulations of the stable distance of two defects for $\omega=0.1$ and $\mu=4$ for various $q$. Stable pulses consisting of two walls exist for $q>0.8$. This location is shown by the box symbol in Fig. 4. The position of the critical $q$ close to the upper zero discriminant line suggests that all four eigenvalues must be complex to allow bound propagating kinks.

In conclusion, we have shown that traveling-stripe forcing produces kinks with dynamical behaviors which differ fundamentally from those of a Hopf unstable system under oscillatory forcing. With no spatial mismatch (and thus in the oscillatory case) traveling kinks emerge in a spontaneous symmetry breaking of Ising kinks. With $q \neq 0$ the spatial reflection symmetry is broken externally. This leads to the occurrence of kinks of preferred chirality and, below the kink saddle node, to the motion of kinks in a preferred direction. Remarkably, this perturbation also generates complex kink interaction and thus chaotic wave trains

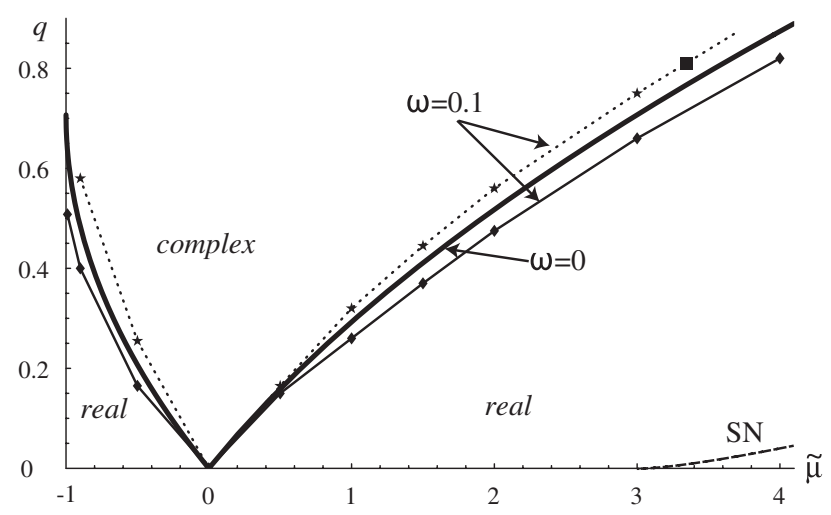

FIG. 4. Complex asymptotic eigenvalues exist above the bold line for $\omega=0$. For $\omega=0.1$ the thin solid and the dotted lines show the location of zero discriminant (numerical convergence was not sufficient for the dotted line close to $\tilde{\mu}=-1$ ). The dashed line represents the kink saddle node for $\omega=0$. 


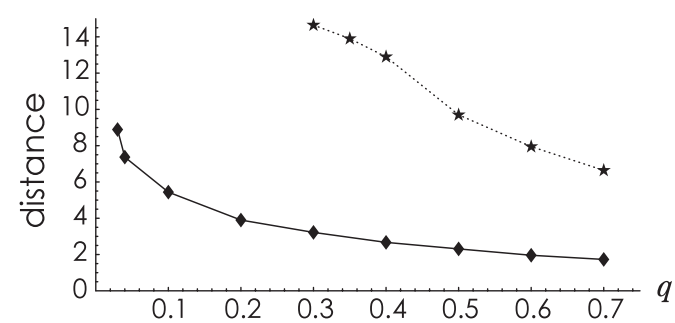

FIG. 5. The stable distance of kinks (stars) and the critical distance for repulsion (diamonds) for $\mu=1$ and $\omega=0$. The transition to oscillating wall interaction occurs at $q \approx 0.28$.

[22]. We have shown that drift of the forcing affects the kink interaction by uncoupling eigenvalues with positive and negative real parts and shifting the location of kink "gluing" in parameter space. This transition is an interesting point of contact to dynamical systems theory, which requires different modulus of eigenvalue real parts for chaotic orbits [27] and could be crucial for existence and stability arguments on chaotic solutions in our system. Similar behavior is expected for different resonances, such as $1: 1$.

We have tested our findings in a mathematical model for the chlorine dioxide-iodine-malonic acid reaction, which exhibits Turing patterns and allows for a spatiotemporal forcing through illumination. Numerical simulations demonstrated the stability of walls for a spatial resonance close to 2:1 [28] (also see online material [26]). Parameter values were chosen to reproduce experimental conditions. This system is thus very adequate to test our predictions experimentally and to explore the problem in $2 d$. Other systems in hydrodynamics, nonlinear chemistry, liquid crystals, or nonlinear optics could also probe the predictions and possibly enrich the scenarios by including effects such as linear and nonlinear dispersion through traveling forcing of a traveling wave. Finally, the control of patterns is a possible use of our study. Spatiotemporal forcing provides a natural tool for defect control or for displacement of nontrivial locked states. These aspects open new possibilities in the context of the recently proposed mechanisms of information storage and transmission in nonequilibrium media [14], with practical applications for instance in nonlinear optics [15] or reaction-diffusion systems [16].

S. R. and J.C. acknowledge support, respectively, from Deutsche Forschungsgemeinschaft (Grant No. FA 350/6-1) and Ministerio de Ciencia y Tecnología, Spain (Project No. FIS2006-03525).

[1] V. Petrov, Q. Oyang, and H. L. Swinney, Nature (London) 388, 655 (1997).

[2] M. Dolnik et al., Phys. Rev. Lett. 87, 238301 (2001).

[3] A. S. Mikhailov and K. Showalter, Phys. Rep. 425, 79 (2006).
[4] M. Lowe, J. P. Gollub, and T. C. Lubensky, Phys. Rev. Lett. 51, 786 (1983).

[5] P. Coullet, Phys. Rev. Lett. 56, 724 (1986).

[6] J.H. McCoy, Ph.D. thesis, Cornell University, 2007; J. H. McCoy, W. Brunner, W. Pesch, and E. Bodenschatz (to be published).

[7] S. Rüdiger, D. G. Míguez, A. P. Muñuzuri, F. Sagués, and J. Casademunt, Phys. Rev. Lett. 90, 128301 (2003).

[8] D. G. Míguez, E. M. Nicola, A. P. Muñuzuri, J. Casademunt, F. Sagués, and L. Kramer, Phys. Rev. Lett. 93, 048303 (2004).

[9] F. Sagués, D. G. Míguez, E. M. Nicola, A. P. Muñuzuri, J. Casademunt, and L. Kramer, Physica (Amsterdam) 199D, 235 (2004).

[10] R. Peter et al., Phys. Rev. E 71, 046212 (2005).

[11] S. Rüdiger, J. Casademunt, and L. Kramer, Discr. Cont. Dyn. Syst. B 5, 1027 (2005).

[12] T. Ohta and H. Tokuda, Phys. Rev. E 72, 046216 (2005).

[13] S. Zykov, V.S. Zykov, and V. Davydov, Europhys. Lett. 73, 335 (2006).

[14] P. Coullet, C. Riera, and C. Tresser, Chaos 14, 193 (2004).

[15] S. Barland et al., Nature (London) 419, 699 (2002).

[16] A. Kaminaga, V. K. Vanag, and I. R. Epstein, Angew. Chem., Int. Ed. 45, 3087 (2006).

[17] P. Coullet et al., Phys. Rev. Lett. 65, 1352 (1990).

[18] A. Hagberg and E. Meron, Phys. Rev. Lett. 72, 2494 (1994).

[19] T. Frisch et al., Phys. Rev. Lett. 72, 1471 (1994).

[20] T. Kawagishi, T. Mizuguchi, and M. Sano, Phys. Rev. Lett. 75, 3768 (1995).

[21] A. Esteban-Martín et al., Phys. Rev. Lett. 94, 223903 (2005).

[22] P. Coullet, C. Elphick, and D. Repaux, Phys. Rev. Lett. 58, 431 (1987).

[23] D. V. Skryabin et al., Phys. Rev. E 64, 056618 (2001).

[24] E. J. Doedel et al., AUTO97 (with HOMCONT), manual (Concordia University, Montreal, 1997); B. Ermentrout, Simulating, Analyzing, and Animating Dynamical Systems: A Guide to XPPAUT for Researchers and Students (SIAM, Philadelphia, 2002).

[25] HOMCONT calculates heteroclinic orbits by approximating them by orbits of a given integration interval. For our calculations we have used orbits with a pseudotime interval of at least 40 .

[26] See EPAPS Document No. E-PRLTAO-98-025726 for supplementary figures and discussion. For more information on EPAPS, see http://www.aip.org/pubservs/epaps. html.

[27] Y. A. Kuznetsov, Elements of Applied Bifurcation Theory (Springer, New York, 1998).

[28] The simulations were performed in a periodic domain for the Lengyel-Epstein model [29], modified to include the effect of illumination [30]. In a domain of around 70 stripes we checked the stability of bound pairs of kinks with and without motion of the forcing. We estimated the critical forcing velocity where kinks disappear. Stability of kinks in a $2 d$ domain was also found.

[29] I. Lengyel and I. R. Epstein, Science 251, 650 (1991).

[30] A.P. Muñuzuri et al., J. Am. Chem. Soc. 121, 8065 (1999). 